\title{
Direct BRLF1 binding is required for cooperative BZLF1/BRLF1 activation of the Epstein - Barr virus early promoter, BMRF1
}

\author{
E.Byrd Quinlivan ${ }^{1,2}$, Elizabeth A.Holley-Guthrie ${ }^{2}$, Melanie Norris ${ }^{2}$, David Gutsch ${ }^{1,2}$, \\ S.L.Bachenheimer ${ }^{2,3}$ and Shannon C.Kenney ${ }^{1,2, *}$ \\ ${ }^{1}$ Department of Medicine, ${ }^{2}$ Lineberger Comprehensive Cancer Center and ${ }^{3}$ Department of \\ Microbiology and Immunology, University of North Carolina at Chapel Hill, Chapel Hill, NC 27599, \\ USA
}

Received June 1, 1992; Revised and Accepted March 5, 1993

\begin{abstract}
Disruption of Epstein-Barr virus (EBV) latency is mediated through the activation of the viral immediateearly proteins, BZLF1 (Z) and BRLF1 (R).i.; (ChevallierGreco, A., et al., (1986) EMBO J., 5, 3243-9; Countryman, and Miller, G. (1985) Proc. Natl. Acad. Sci. USA, 82, 4085-4089). We have previously demonstrated that these proteins cooperatively activate the EBV early promoter BMRF1 in lymphoid cells but not in epithelial cells. Although cooperative transactivation by these proteins has been demonstrated with a number of EBV promoters, the mechanism of this interaction is not well understood. We now show that the cooperative activation of the BMRF1 promoter by Z-plus- $R$ requires an intact $R$ binding site and at least one functional $Z$ response element (ZRE). Despite the presence of an $R$ binding site, the BMRF1 promoter is only moderately responsive to $\mathbf{R}$ alone in either HeLa or Jurkat cells. Efficient activation of the BMRF1 promoter by $Z$ alone in HeLa cells requires two ZREs (located at -59 and - 106), whereas two additional $Z$ binding sites (located at -42 and -170 ) contribute very little to $Z$-induced activation. In the absence of ZREs, $Z$ acted as a repressor of $R$-induced transactivation. These observations, along with observations made by other investigators (Giot, J.F. et al., (1991) Nucleic Acids Res., 19, 1251 - 8), suggest that Z-plus-R cooperative activation is dependent upon 1) direct binding by $R$ and $Z$ to responsive promoter elements and 2) contributions by cell-specific factors.
\end{abstract}

\section{INTRODUCTION}

Epstein-Barr virus (EBV) produces either a latent (predominantly in B cells) or lytic (predominantly in epithelial cells) infection $(1,2)$. Overexpression of the immediate-early BZLF1 transactivator $(Z)$ is sufficient for reactivation of EBV infection from latency $(3-10)$, indicating that $Z$ plays a key role in EBV regulation. Z-induced activation is mediated by direct binding of the $\mathrm{Z}$ homodimer to specific DNA sequence motifs, including consensus AP-1 and C/EBP sites as well as $\mathrm{Z}$ response elements (ZREs) (11-17). A second EBV immediate-early protein, BRLF1 (R), (18) is translated from a bicistronic mRNA which also encodes $Z$ (19). Consistent with the notion that $R$ and $\mathrm{Z}$ have cooperative functions during viral reactivation in vivo, several EBV early promoters have been shown to be cooperatively activated by $\mathrm{R}$ and $\mathrm{Z}$ in transient expression assays $(13,20,21,25)$. $R$ has recently been reported to be a sequencespecific DNA-binding protein $(21-24)$. However, at present only one laboratory has been able to demonstrate R DNA binding activity (23) and very little is known about the characteristics of the $R$ response elements. The importance of binding by $R$ in $\mathrm{Z}$-plus-R cooperative activation of responsive promoters has not been well studied.

We have previously found that $\mathrm{R}$ and $\mathrm{Z}$ interact cooperatively to activate the early BMRF1 promoter in lymphoid cells but not in epithelial cells and that the cooperative effect of Z-plus-R occurs at the level of BMRF1-directed transcription (25). We have shown that the $\mathrm{Z}$ protein binds to a consensus AP-1 site in the BMRF1 promoter (26), but other potential $\mathrm{Z}$ or $\mathrm{R}$ binding sites in the BMRF1 promoter have not been previously studied.

In this paper, we describe experiments which extend our previous work on the regulation of the BMRF1 promoter by $\mathrm{Z}$ and $R$. We demonstrate that transactivation of the BMRF1 promoter by $\mathrm{Z}$ in HeLa cells is mediated by direct $\mathrm{Z}$ binding to the AP-1 site and an additional upstream $Z$ response element. We show that the $R$ transactivator also binds directly to the BMRF1 promoter and that both the $\mathrm{R}$ binding site and one of the functional ZREs are required for efficient Z-plus-R activation. In addition, we demonstrate that $\mathrm{Z}$ can function as either an enhancer or repressor of $R$-induced transactivation depending upon the presence or absence of functional $\mathrm{Z}$ binding sites. These results are in agreement with recent findings by another laboratory using artificial promoter constructs (21) and suggest that

* To whom correspondence should be addressed 
cooperative transactivation of the BMRF1 promoter by the Zplus- $R$ combination is mediated by direct binding by both the $\mathrm{Z}$ and $\mathrm{R}$ transactivators to responsive promoter elements. The increased activation which is seen when both $\mathrm{R}$ and $\mathrm{Z}$ bind to this promoter could result directly from the cooperative effects of the $\mathrm{Z}$ and $\mathrm{R}$ transactivator domains (27). However the finding that cooperative activation of BMRF1 promoter does not occur in epithelial cells suggests that cellular factors must contribute to Z-plus-R cooperative activation.

\section{MATERIALS AND METHODS Cell lines}

Jurkat, a T-cell line, was maintained in RPMI 1640 medium; $\mathrm{HeLa}$, a cervical epithelial cell line, was maintained in Dulbecco modified Eagle medium $\mathrm{H}$. Both were supplemented with $10 \%$ fetal calf serum.

\section{DNA transfections}

Plasmid DNA was purified through two sequential cesium chloride gradients. DNA was transfected into both lymphoid and epithelial cell lines by electroporation (28). For each condition, $10^{7}$ cells were shocked at $1,500 \mathrm{~V}$ with the Zapper electroporation unit (Medical Electronics Shop, University of Wisconsin). Epithelial cells were harvested and resuspended into RPMI medium for electroporation.

\section{Plasmids}

The plasmid pEA-BS-CAT was made by inserting the $X b a \mathrm{I}-$ BamHI fragment of pEA-CAT (which contains the complete BMRF1 promoter sequences, 79,537 to 79,898 , the CAT gene and the SV40 polyadenylation site) into the XbaI-BamHI site of the M13 Bluescript SK+ plasmid (Stratagene), as previously reported (25). The pEBV-ZIE plasmid contains the BamHI Z gene fragment (EBV nucleotides 101,741 to 103,947$)$ in the pGEM2-based vector pHD1013 (29), such that the BZLF1 gene is under the control of the strong human cytomegalovirus (CMV) immediate-early promoter. In the $\mathrm{pEBV}$ RIE plasmid, a HindIII - HindIII fragment from pEBV-RZ (which contains EBV nucleotides 103,080 to 105,412 linked to the CMV promoter) (25) was ligated into the pUC18 vector.

\section{CAT assays}

Cells were transfected with $10 \mu \mathrm{g}$ of plasmid DNA $(5 \mu \mathrm{g}$ transactivator and $5 \mu \mathrm{g}$ reporter construct) as described above. At 48 hours after transfection, a cell extract was prepared and incubated at $37^{\circ} \mathrm{C}$ with ${ }^{14} \mathrm{C}$ chloramphenicol in the presence of acetyl coenzyme A as described previously (30). The percent acetylation of chloramphenicol was quantitated by thin layer chromatography followed by scanning of the chromatography plates by an AMBIS scanner (AMBIS, Inc.) for 10 hours. If results were not in the linear range, CAT assays were repeated with less extract.

\section{In vitro site-directed mutagenesis}

Mutants were made by using the BIO-Rad Muta-Gene phagemid in vitro mutagenesis kit, based on a method described by Kunkel et al. (31). Synthetic oligonucleotides were complementary to the region to be altered except for a limited internal mismatch. For screening possible mutants, synthetic oligonucleotides were created such that in vitro mutations would create a new PvuII or $\mathrm{XbaI}$ restriction site allowing each possible mutant to be analyzed by digestion with restriction endonucleases. Following initial screening, potential mutants were sequenced with the Sequenase 2.0 enzyme sequencing kit (United States Biochemical Corp.). Single stranded oligomers used for constructing sitedirected mutants are listed below. Mutated sequences within the oligomers are indicated by underlining.

-106ss: TGTAATCGGAACTCTCTCTAGAAAAGAAATTAGT
AP1ss: GTAGCCACCCTCAGCTGAAGGTCATCTACC
-42ss: GCCGAGAAACCTGACAGCTGAGCCACCCTG
-158ss: GTAGGTGAGCGGGCTCTAGATGGGGGGTGG
-170ss: TGGGGGGTCTAGACCATACAAGGGAGCCT
-182ss: TGTGCCATACAATCTAGACTGACCAATGTC

\section{$\mathbf{Z}$ protein expression}

The in vitro translated BZLF1 protein was expressed from an SP64 vector containing an 870-base-pair cDNA for BZLF1 (gift of P.Farrell, (11)). The plasmid was digested with EcoRI and transcribed using SP6 polymerase (Promega). The RNA obtained was used to program protein synthesis using wheat germ extract (Promega). Correct translation was confirmed using ${ }^{35} \mathrm{~S}$ methionine-labeled protein analyzed by SDS-PAGE and autoradiography.

A Z/glutathione S-transferase fusion protein (GST-Z) was created by removing the Z cDNA from the SP64 construct (gift of P.Farrell, (11)) with the restriction enzymes BamHI and EcoRI, filling in all $5^{\prime}$ protruding ends with Klenow DNA polymerase and ligating to the SmaI site of the glutathione Stransferase (GST) vector, pGEX-3X. This put the BZLF1 sequence in frame, and downstream, of the glutathione Stransferase carrier protein to create the plasmid pGST-Z. In the E.coli host $\mathrm{DH} 5 \alpha \mathrm{F}^{\prime}$, these plasmids were induced for 4 hours at $30^{\circ} \mathrm{C}$ with $1 \mathrm{mM}$ IPTG. Bacteria were lysed in $50 \mathrm{mM} \mathrm{KCl}$, $8 \mu \mathrm{M}$ leupeptin and $0.5 \mathrm{mM}$ PMSF by sonication, and cleared by centrifugation. Lysates were assayed for protein concentration by the Bradford method (32), then used directly in gel retardation assays.

\section{$\mathbf{R}$ protein expression}

Using the polymerase chain reaction (PCR) with Vent polymerase (New England Biolabs), an EcoRI site was created just $5^{\prime}$ to the BRLF1 initiation codon. An EcoRI to ApaI fragment of the PCR product, containing the first 356 codons of the BRLF1 reading frame, was ligated blunt end into the EcoRI site of the pGEX-3X expression vector (Pharmacia) by first filling in all $5^{\prime}$ protruding ends with Klenow DNA polymerase. This put the BRLF1 sequence in frame, and downstream, of the glutathione Stransferase carrier protein to create the plasmid pGR356 encoding the GST carrier protein fused to the first 356 amino acids of $R$ (GR356). The negative control vector, $\mathrm{pGR}-$, contains the BRLF1 reading frame in an antisense orientation with respect to glutathione S-transferase. In the E.coli host DH5 $\alpha \mathrm{F}^{\prime}$, these plasmids were induced for 4 hours at $30^{\circ} \mathrm{C}$ with $1 \mathrm{mM}$ IPTG. Bacteria were lysed in $50 \mathrm{mM} \mathrm{KCl}, 8 \mu \mathrm{M}$ leupeptin and $0.5 \mathrm{mM}$ PMSF by sonication, and cleared by centrifugation. Lysates were assayed for protein concentration by the Bradford method (32), then used directly in gel retardation assays.

\section{Gel retardation assays}

DNA binding reactions were performed as described previously $(11,23)$ with the following modifications. ${ }^{32} \mathrm{P}$-labeled probes were constructed by isolating restriction fragments of the BMRF1 promoter. $5 \mu \mathrm{l}$ of in vitro translated $\mathrm{Z}$ protein, $5 \mu \mathrm{g}$ of GST-Z 
fusion protein or GR356 fusion protein (or equal amounts of the corresponding negative control) were incubated with $2 \times 10^{4}$ cpm of ${ }^{32} \mathrm{P}$-labeled probes for 30 minutes at room temperature. The $\mathrm{Z}$ binding reactions were performed in $20 \mu \mathrm{l}$ of $100 \mathrm{mM}$ $\mathrm{KCl}, 20 \mathrm{mM}$ HEPES (pH 7.3), 10\% glycerol, $0.2 \mathrm{mM}$ EDTA, $4 \mathrm{mM}$ dithiothreitol, $0.50 \mathrm{mM}$ PMSF with $2 \mu \mathrm{g}$ of poly (dIdC - dIdC). The R binding reactions were performed in $30 \mu \mathrm{l}$ of $130 \mathrm{mM} \mathrm{KCl}, 10 \mathrm{mM}$ HEPES (pH 7.3), 5\% Ficoll, $5 \mathrm{mM} \mathrm{MgCl}_{2}, 0.5 \mathrm{mM}$ dithiothreitol, $0.5 \mathrm{mM}$ PMSF with $1 \mu \mathrm{g}$ of poly ( $\mathrm{dIdC} \cdot \mathrm{dIdC}$ ) at $4^{\circ} \mathrm{C}$. The retarded complexes were detected by loading the entire sample onto a $5 \%$ polyacrylamide gel, run in $0.5 \%$ TBE buffer and detected by autoradiography.

The synthetic double-stranded oligonucleotides used in binding reactions are listed below. Mutated sequences within the oligomers are indicated by underlining.

RRE-BMLF1 (EBV 84,696-84,735): AGATGTCCCTCTATCATGGCGCAGACATTCTCCACGGTGG

MYB $_{\text {RE4: }}$ CCGAATTCCAGTTAATAGCAGTTAATAGCAGTTAATAGCAGTTAATTT

AP-1 (EBV 79,801-79,821): ACCTTTGAGTCAGGGTGGCTA

RRE-DR (EBV 53,524-53,552): TGGGAATTCCCTGTGCCTTGTCCCGTGGACAATGTCCCCCGGGTCC
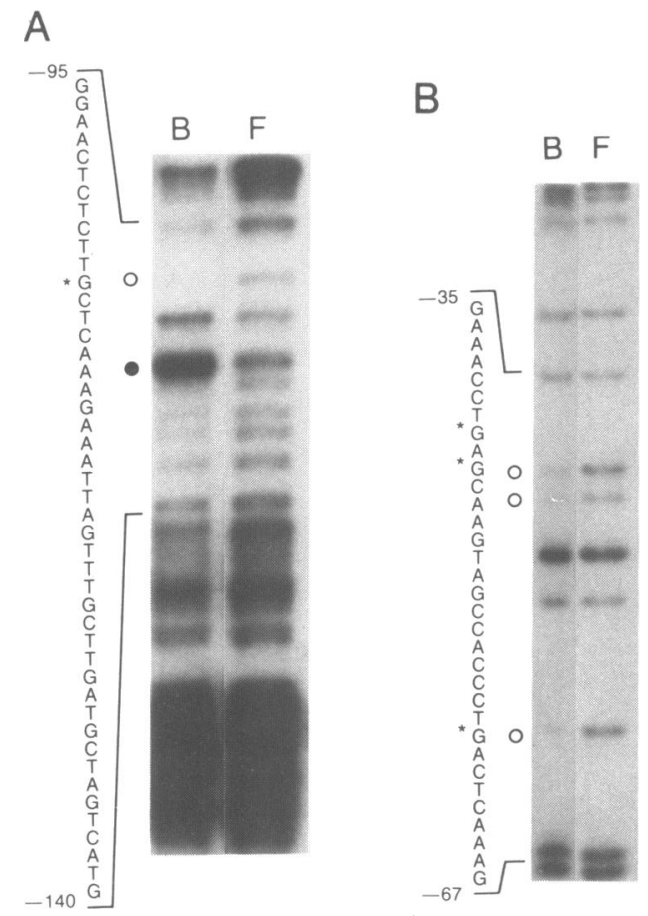

Figure 1. Identification of $Z$ binding sites within the BMRF1 promoter. A. ${ }^{32-P}$ labeled probe containing BMRF1 sequences from -79 to -265 was incubated with in vitro translated $\mathrm{Z}$ protein and then treated with dimethylsulfate immediately prior to gel electrophoresis. The bands representing bound (B) and free (F) probe were removed separately, cut with piperidine, and analyzed on an $8 \%$ polyacrylamide sequencing gel. The sequence in the region of protection is shown. Areas of protection are indicated $(0, *)$ and an area of hypermethylation is indicated $(\bullet)$. B. Probe containing the BMRF1 sequences from -79 to +125 . C. Gel retardation assay performed with GST-Z fusion protein and a probe spanning the -265 to -79 region of the wildtype BMRF1 promoter. The protein and the double-stranded oligomer spanning the -153 to -187 region of the BMRF1 promoter used as competitor DNA at 1000 molar excess are indicated above each lane. (GST: glutathione S-transferase protein; Z: GST-Z fusion protein; WT: wildtype RRE-BMRF1 oligomer; $\Delta-158$ : BMRF $1 \Delta-158$ oligomer; $\Delta-170$ : BMRF1 $\Delta-170$ oligomer; $\Delta-182$ : BMRF1 $\Delta-182$ oligomer.)
RRE- $\triangle D R$ (EBV 53,524-53,552): TGGGAATTCCCTGTGCCTTGTCCCGTGTCAAATCAACCCCGGGTCC

RRE-BMRF1 (EBV 79,683-79,717): GATCGCTCCCTTGTATGGCACACCACCCCCCAAGGACTGGATC

BMRF1 $\Delta-158$ (EBV 79,683-79,717): GATCGCTCCCTTGTATGGCACACCACCCCCCATCTAGAGGATC

BMRF1 $\triangle-170$ (EBV 79,683-79,717): GATCGCTCCCTTGTATGGTCTAGAACCCCCCAAGGACTGGATC

BMRF1 18 - 182 (EBV 79,683-79,717): GATCTCTAGATTGTATGGCACACCACCCCCCAAGGACTGGATC

\section{Methylation interference and protection assays}

Methylation interference was performed as previously described (33). $1 \times 10^{5} \mathrm{cpm}$ of methylated probe was incubated with 10 $\mu \mathrm{g}$ of GR356 fusion protein in two volumes of the binding reaction mixture used for gel retardation assays described above. The bound and free species were excised from a $5 \%$ polyacrylamide gel and electroeluted onto DEAE membrane paper (Schleicher and Schuell). The membrane was incubated with $1 \mathrm{M}$ piperidine at $90^{\circ} \mathrm{C}$ for $15-30$ minutes, then rinsed twice with $1 \mathrm{ml}$ of sterile water. The probe fragments were then eluted by incubating the membrane in 250 ul of $1 \mathrm{M} \mathrm{NaCl}, 0.1 \mathrm{mM}$ EDTA and $20 \mathrm{mM}$ Tris $(\mathrm{pH} 8)$ at $67^{\circ} \mathrm{C}$ twice and ethanol precipitated. Dried samples were resuspended in formamide buffer, loaded onto either a $6 \%$ or a $8 \%$ polyacrylamide- $7 \mathrm{M}$ urea sequencing gel and visualized by autoradiography. Methylation protection was performed by modifying the methylation interference procedure. Unmethylated probe was incubated with $10 \mu \mathrm{l}$ of in vitro translated $\mathrm{Z}$ protein or $10 \mu \mathrm{g}$ GR356 fusion protein in two volumes of binding reaction mixture. $1 \mu \mathrm{l}$ of dimethylsulfate was added to the binding reaction immediately prior to loading onto a $5 \%$ polyacrylamide, $0.5 \%$ TBE gel. Isolation of the bound and free complexes, cleavage with piperidine and elution of the probe fragments were performed as described above.

\section{RESULTS}

We have previously shown that the BMRF1 promoter responds to the $\mathrm{Z}$ transactivator alone in HeLa cells and to the $\mathrm{R}$ and $\mathrm{Z}$ transactivators cooperatively in Jurkat cells (25). We have also shown that the Z-induced activation of the BMRF1 promoter is mediated by direct binding to the consensus AP-1 site (26). In this study we extended our examination of $\mathrm{Z}$ binding to the BMRF1 promoter and explored the role of $R$ and $Z$ binding sites in activation of the BMRF1 promoter by $R$ and $Z$.

\section{$Z$ response elements}

To identify other potential $\mathrm{Z}$ binding sites in the BMRF1 promoter, we performed methylation protection assays (33) using in vitro translated $\mathrm{Z}$ protein. A methylation protection experiment (Fig. 1A) using a probe containing the -265 to -79 region of the BMRF1 promoter showed protection of the guanosine at -106 (EBV 79,764). The sequence in this region is TTGCTCA which is homologous to a previously described $\mathrm{Z}$ binding site (ZRE2) in the BHLF1 promoter (15). Weak protection was also seen at $-128,-131$, and -135 . This sequence bears no clear homology to previously described $\mathrm{Z}$ binding sites.

Using a probe (Fig. 1B) containing the region -79 to +125 of the BMRF1 promoter, we observed protection over the AP-1 site as previously reported (26). Additionally, in some experiments we observed protection at residues -42 and -44 . 
The sequence in this region, TGAGCAA, is a one basepair mismatch of both the ZIIIA site in the BZLF1 promoter (12, 17) and the ZRE5 site in the BHLF1 promoter (15). Taylor et al. have reported that an oligomer containing the TGAGCAA sequence bound $\mathrm{Z}$ efficiently in a gel retardation assay (17).

A.

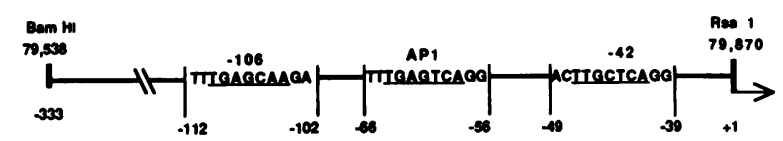

B. PLASMIDS

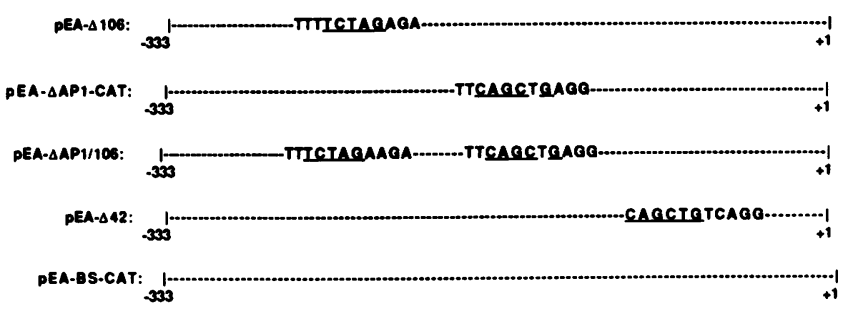

Figure $2 \mathrm{~A}$. Map of the BMRF1 promoter. The $\mathrm{Z}$ binding sites of the BMRF1 promoter are shown. B. Construction of pEA-BS-CAT ZRE site-directed mutants. The parent construct, pEA-BS-CAT, contains the EBV BamHI-RsaI fragment linked to CAT in a phagemid vector (25). The $\mathrm{Z}$ binding sites were abolished using the Kunkel method (31) of site-directed mutagenesis as shown.
An additional $\mathrm{Z}$ binding site was found during our studies on $\mathrm{R}$ binding with the BMRF1 promoter. This site was too weak to be identified by methylation protection but it was able to bind to $\mathrm{Z}$ in a gel retardation assay (Fig. 1C). This site was defined by using as competitors a series of double-stranded mutant oligomers which span the -187 to -153 region of the BMRF1 promoter. The oligomer containing a mutation which disrupted the sequence TGGCACA $(\Delta-170)$ was no longer able to compete successfully for $Z$ binding (Fig. 1C, Lane 5). This site is a one basepair mismatch with the ZIIIA site in the $\mathrm{Z}$ promoter (17) as well as a number of other $Z$ binding sites $(13,15)$.

The functional importance of the four $\mathrm{Z}$ binding sites in the BMRF1 promoter was defined by cotransfection and chloramphenicol acetyltransferase (CAT) $(28,30)$ assays in HeLa and Jurkat cells. Using the method of Kunkel et al. (31), we constructed mutations abolishing the -170 site, -106 site, the AP-1 site (25), the -42 site or the -106 and AP-1 sites together (Fig. 2). In HeLa cells, where $\mathrm{Z}$ alone is sufficient to induce maximal transactivation of the BMRF1 promoter (25), deletion of either the -106 site or the AP-1 site significantly decreased $\mathrm{Z}$ responsiveness (Fig. 3A). Only $13 \%$ of the $\mathrm{Z}$-induced activity of the intact BMRF1 promoter was observed after removing the upstream binding site and only $9 \%$ of the activity of the intact BMRF1 promoter was seen after removing the AP-1 site. When both sites were removed $(\Delta \mathrm{AP} 1 /-106), \mathrm{Z}$ response was further decreased to $3 \%$ of the wildtype promoter. Mutation of the -42 site $(\Delta-42)$ or the -170 site $(\Delta-170)$ had no effect on $\mathrm{Z}$ transactivation. Therefore, in HeLa cells, only two of the four

\section{A}
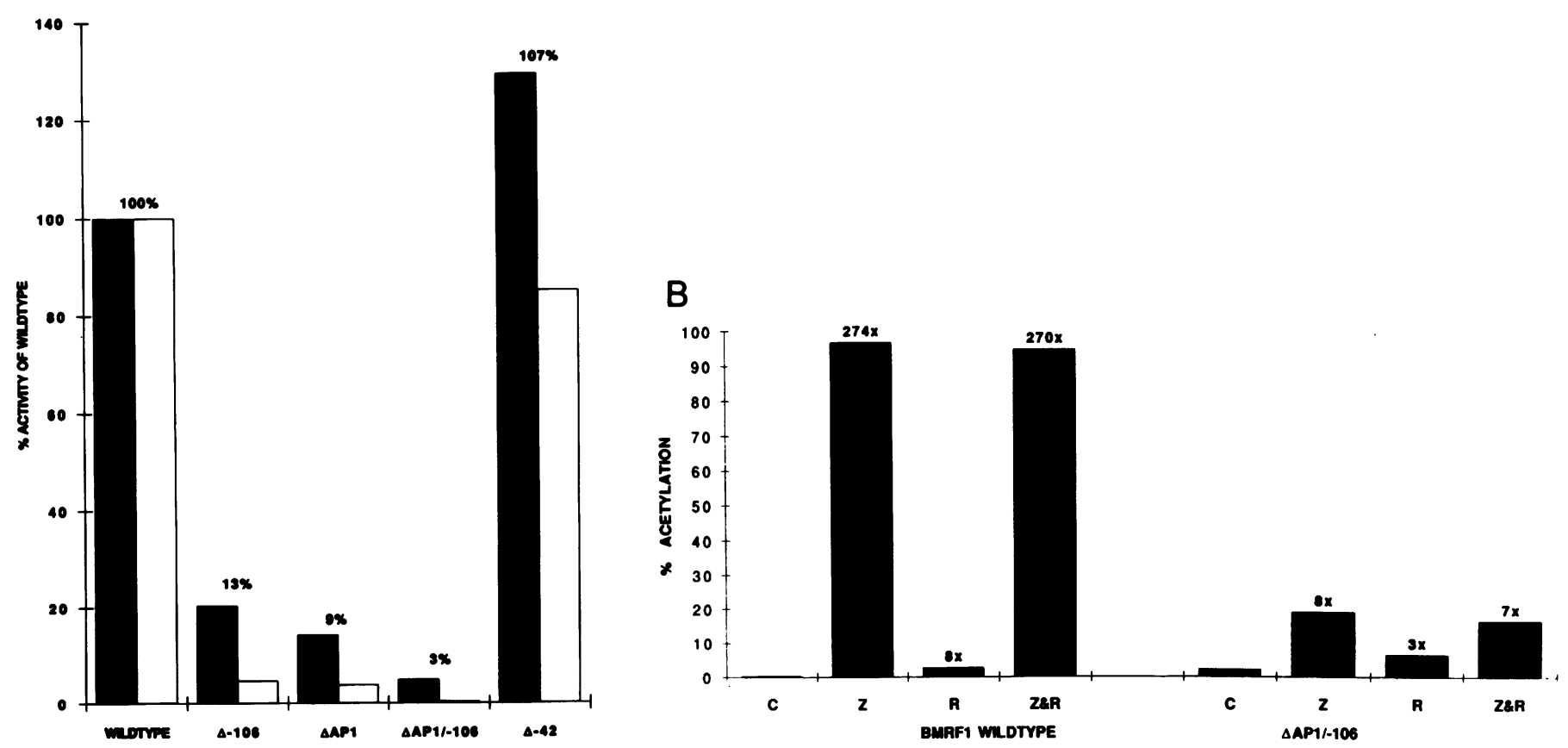

Figure 3A. The BMRF1 promoter requires two $Z$ binding sites for $Z$ activation in HeLa cells. The BMRF1 promoter constructs (Fig. 2B) pEA-BS-CAT (wildtype), pEA- $\Delta 106(\Delta-106)$, pEA- $\Delta$ AP-1-CAT $(\Delta$ AP1), pEA- $\Delta$ AP1/106 $(\Delta$ AP1/-106) pEA- $\Delta 42(\Delta-42)$, or pEA- $\Delta 170(\Delta-170)$ were cotransfected with pEBV-ZIE (Z) into HeLa cells. The results are presented as average percent of the activity of the various promoter constructs plus $Z$ versus the intact promoter (wildtype) plus $Z$ from two or three separate experiments. The upper range of the experiments is indicated by the bar above the columns. $B$. The $Z$ and $R$ transactivators do not cooperatively activate the BMRF1 promoter in HeLa cells. The BMRF1 promoter constructs pEA-BS-CAT (wildtype), or pEA- $\triangle \mathrm{AP} 1 / 106(\Delta \mathrm{AP} 1 /-106)$ were cotransfected with pHD1013 (C), pEBV-ZIE (Z), pEBV-RIE (R) or both pEBV-ZIE and pEBV-RIE (Z\&R) into HeLa cells. The results are presented as average percent acetylation of two experiments (indicated in the ordinate). The numbers above the bars indicate the average fold induction by the transactivator versus the control plasmid. 
$\mathrm{Z}$ binding sites ( -106 and AP-1) appear to be functional ZREs and both are required for efficient $Z$ transactivation. After destruction of both the -106 and AP-1 sites, $R$ remained unable to function cooperatively with $\mathrm{Z}$ in this cell type (Fig. 3B).

The importance of each $\mathrm{Z}$ binding site was also determined in Jurkat cells, where maximal activation of the BMRF1 promoter requires the combination of Z-plus-R (25) (Fig. 4). Loss of either the AP-1 site alone or the upstream -106 site alone did not abolish the ability of the BMRF1 promoter to respond to the Zplus-R combination. However, deletion of either ZRE increased the constitutive activity of the BMRF1 promoter, resulting in a reduction in the fold-increase produced by the Z-plus-R combination. This increase in constitutive activity was particularly striking when both the -106 and AP-1 sites were deleted simultaneously. Destruction of the -42 site had only a minimal effect on Z-plus-R activation. The effects of destroying the -170 site are shown in Fig. 9. This mutation disrupts Z-plus-R cooperative activation but as we show below it also disrupts an $\mathrm{R}$ binding site. Thus it appears that only the AP-1 and -106 sites actually function as ZREs.

When both ZREs were abolished ( $\triangle \mathrm{AP} 1 /-106)$, the BMRF1 promoter no longer responded to the Z-plus-R combination. Of note, however, was the finding that $R$ alone transactivated the double mutant approximately 4 -fold, compared to the 0.7 fold effect induced by $Z$ and $R$ together. Thus, in the absence of $\mathrm{ZREs}, \mathrm{Z}$ appeared to inhibit the moderate $\mathrm{R}$-induced activation of the BMRF1 promoter.

\section{$R$ response elements}

To determine whether the cooperative effect of Z-plus-R on BMRF1 promoter activity might also be mediated by direct $R$ binding to the promoter, we created an $\mathrm{R} /$ glutathione $\mathrm{S}$ - transferase fusion protein (GR356) for protein binding studies. The $\mathbf{R}$ protein has been shown to bind as a homodimer to three sites which consist of two contact regions separated by $6-8$ nucleotides $(22,23)$. We constructed a probe spanning the BMRF1 promoter from -265 to +1 . We were able to demonstrate $\mathrm{R}$ binding to the BMRF1 promoter (Fig. 5) which was specifically competed by oligomers containing known $R$ binding sites from the EBV early DR and BMLF1 promoters (Fig. 5, Lanes 3, 5) $(22,23)$ but not by oligomers containing a mutated DR promoter $\mathrm{R}$ binding site or $c-m y b$ binding sites (Fig. 5, Lane 4, 6) (26).

To identify precisely where $\mathrm{R}$ bound to the BMRF1 promoter, we performed methylation protection and interference assays using the GR356 fusion protein. A methylation protection experiment (Fig. 6A), using a probe labeled on the coding strand and containing the -265 to +1 region of the BMRF1 promoter, showed definite protection of the guanosine at -158 and possibly at -157 (EBV 79,712 and 79,713). The sequence in this region, AGGAC, shows homology ( $4 / 5$ basepair match) to the GGGAC half of the DR-2 R binding site in the DR promoter (23). An extra band does appear in the lane containing the bound probe which may represent a hypermethylated adenosine residue at $-156$.

Using a probe (Fig. 6B) containing the region -265 to -79 of the BMRF1 promoter and labeled on the noncoding strand, we observed strong protection of the guanosines at $-173,-171$, $-169,-168$, and -155 and weak protection of the region from -166 to -161 in a methylation interference assay. The sequence in the region -155 to -171 (GTCCT tggggg GTGGTG) is a one basepair mismatch of the DR-2 R binding site and includes the protected region on the opposite strand (Fig. 7). In addition, there was questionable protection in the -182 region. The

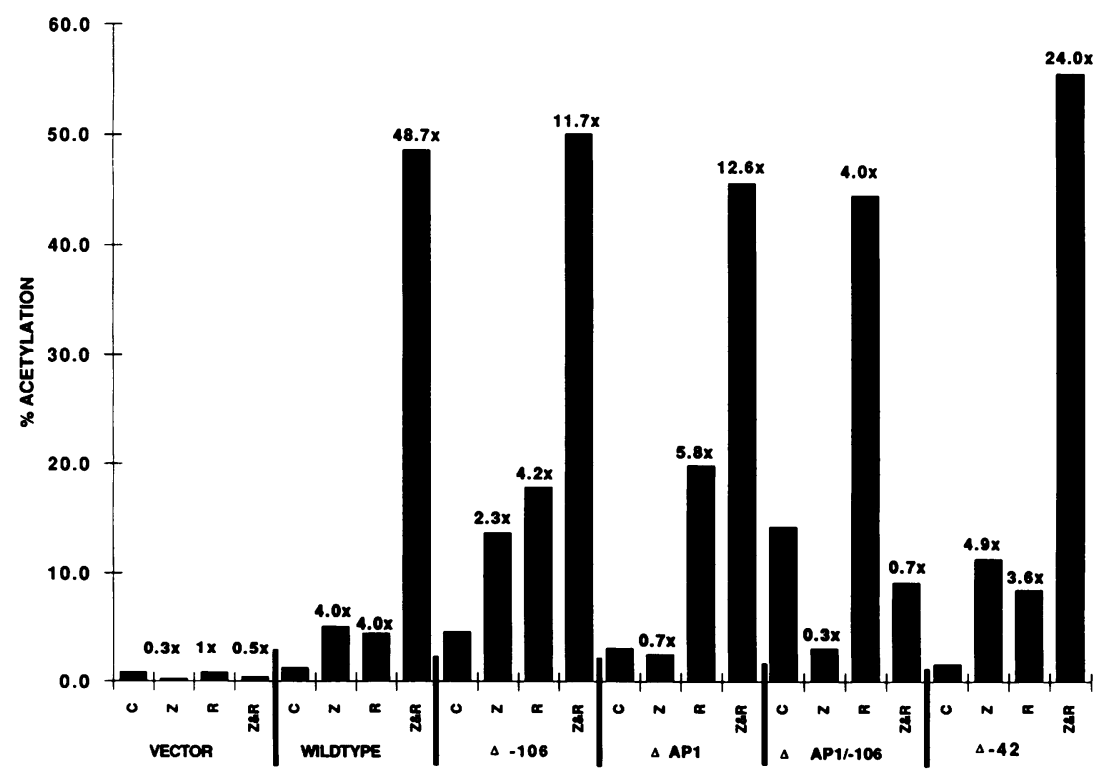

Figure 4. Z-plus-R transactivation of the BMRF1 promoter in Jurkat cells requires at least one ZRE. The CAT constructs pBS-CAT (vector without BMRF1 promoter), pEA-BS-CAT (wildtype), pEA- $\Delta 106(\Delta-106)$, pEA- $\Delta$ AP1-CAT $(\Delta$ AP1), pEA- $\Delta$ AP1/106 $(\Delta$ AP1/ -106$)$ or pEA- $\Delta 42(\Delta-42)$ were cotransfected with a control plasmid (pHD1013, C), pEBV-ZIE (Z), pEBV-RIE (R), or both (Z\&R) into Jurkat cells. The results are presented as average percent acetylation of either one (vector), two $(\Delta-42)$, or three (all others) experiments (indicated in the ordinate). The numbers above the bars indicate the average fold induction by the transactivator versus the control plasmid. The average fold induction was calculated by averaging the fold induction of each individual experiment. 
A.

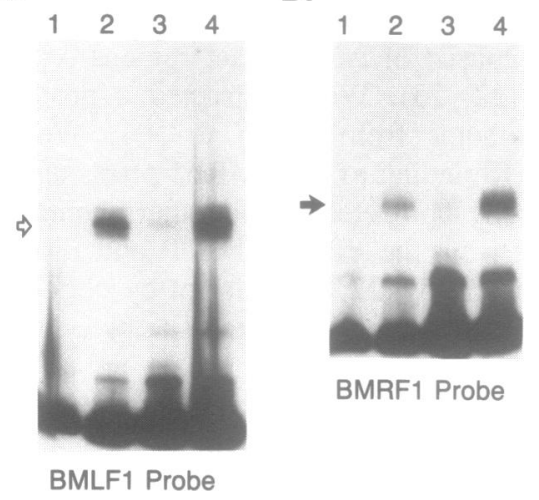

Figure 5. A bacterial $\mathrm{R}$ fusion protein binds to the BMRF1 promoter. A gel retardation assay was performed using a bacterial $R$ fusion protein (GR356, $R$ ) and a probe spanning the BMRF1 promoter from -265 to +1 relative to the mRNA start site. The protein and the double-stranded oligomer used as competitor DNA at 1000 molar excess are indicated above each lane. (GR - : fusion protein constructed with $R$ in the antisense orientation; R: GR356 fusion protein; RREDR: oligomer containing a DR promoter $\mathrm{R}$ binding site; RRE- $\triangle \mathrm{DR}$ : oligomer containing a mutation in the DR promoter $\mathrm{R}$ binding site; RRE-BMLF1: oligomer containing the BMLF1 promoter $\mathrm{R}$ binding site; $\mathrm{MYB}_{\mathrm{RE} 4}$ : oligomer containing four c-myb binding sites.)

sequence in this area $(-186$ to -171$)$ contains limited homology with the DR-1 site (8/11 basepair match with an interval of six basepairs instead of seven, see Fig. 7).

Further definition of the $\mathrm{R}$ binding site(s) was accomplished using gel retardation assays. Again we used the method of Kunkel et al. (31) to construct mutants disrupting the -182 , the -170 or the -158 regions (Fig. 7). Probes spanning the -265 to -79 region were made from the wildtype, the $\Delta-182, \Delta-170$, and $\Delta-158$ BMRF1 promoter constructs. Both the $\Delta-158$ and the $\Delta-170$ constructs failed to bind R (Fig. 8A, Lanes 8, 10) whereas the wildtype and the $\Delta-182$ constructs were able to bind to the GR356 fusion protein (Fig. 8A, Lanes 2, 12). These results were confirmed with a competition study in which doublestranded oligomers containing the wildtype sequence or the three mutant sequences were used as competitors. The wildtype and the $\Delta-182$ mutant oligomers were able to disrupt $\mathrm{R}$ binding (Fig. 8A Lane 5 and Fig. 8B Lanes 3,6) but the $\Delta-158$ and the $\Delta-170$ mutant oligomers were not able to disrupt the R/BMRF1 complex (Fig. 8B Lanes 4, 5).

The functional importance of these sites of protection was defined by using cotransfection and CAT assays in Jurkat cells. In Jurkat cells, where $\mathrm{R}$ functions cooperatively with $\mathrm{Z}$ to produce activation of the BMRF1 promoter, destruction of the -158 region (a mutation which disrupts $\mathrm{R}$ binding) reduced the level of Z-plus-R activation to $30 \%$ of that seen with the wildtype promoter (Fig. 9). Destruction of the -170 region (a mutation which disrupts both $\mathrm{R}$ and $\mathrm{Z}$ binding to this region) reduced the efficiency of Z-plus-R activation only slightly more efficiently. The -182 mutant reduced Z-plus-R activation of the BMRF 1 promoter to $65 \%$ of that seen with the wildtype promoter, a reduction of questionable significance. Thus, an R binding site is located in the BMRF1 promoter spanning the -170 and -158 regions (Fig. 10) and this binding site is important for Z-plus$\mathbf{R}$-induced activation.

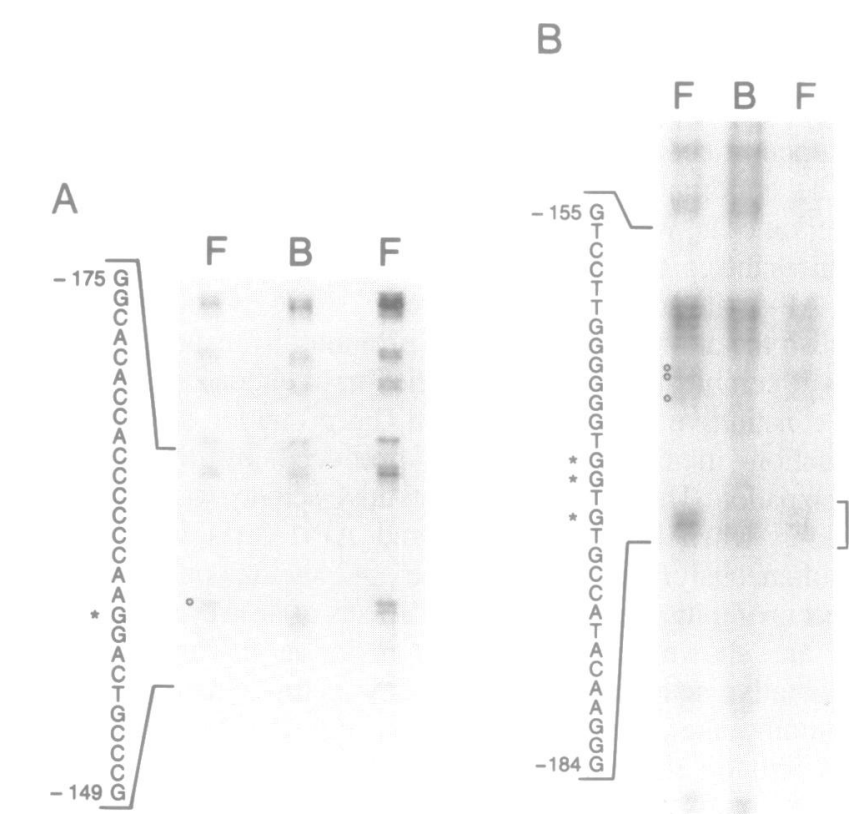

Figure 6. Mapping of the $\mathrm{R}$ binding sites in the BMRF1 promoter. A. A ${ }^{32-\mathrm{P}-}$ labeled probe containing BMRF1 sequences from -265 to +1 (coding strand) was incubated with GR356 fusion protein and then treated with dimethylsulfate immediately prior to gel electrophoresis. The bands representing bound (B) and free $(F)$ probe were removed separately, cut with piperidine, and analyzed on an $8 \%$ polyacrylamide sequencing gel. The sequence in the region of protection is shown. Areas of protection are indicated $\left(0,{ }^{*}\right)$. B. A methylated ${ }^{32-P}$-labeled probe containing BMRF1 sequences from -265 to -79 (noncoding strand) was incubated with GR356 fusion protein. The sequence in the region of protection is shown. Areas of protection are indicated $(0, *)$.

\section{DISCUSSION}

Although a number of EBV promoters are cooperatively activated by the $\mathrm{Z}$-plus- $\mathrm{R}$ combination, the function of $\mathrm{Z}$ and $\mathrm{R}$ binding sites in this interaction has not been carefully analyzed. In this study, we have dissected the role of $\mathrm{Z}$ and $\mathrm{R}$ binding sites in the BMRF1 promoter in regard to activation by $Z$ versus the combination of $Z$-plus- $R$. We have identified two $Z$ binding sites which function as ZREs. The presence of these two elements was shown to be critical for Z-induced activation in HeLa cells, whereas either ZRE alone conferred Z-plus-R responsiveness in Jurkat cells. $\mathrm{R}$ was also shown to bind to the BMRF1 promoter and destruction of the $\mathrm{R}$ binding site reduced the Z-plus-R effect. Therefore, the cooperative transactivation of the BMRF1 promoter by the Z-plus-R combination in Jurkat cells appears to be mediated by direct binding of both the $\mathrm{Z}$ and $\mathrm{R}$ transactivators.

In HeLa cells, $\mathrm{Z}$ is able to transactivate the BMRF1 promoter independently, and we have demonstrated that both the -106 and AP-1 $(-59) \mathrm{Z}$ binding sites are required for $\mathrm{Z}$-induced activation. Carey et al. have recently shown that the presence of multiple $\mathrm{Z}$ binding sites within a promoter contributes to cooperative activation by $\mathrm{Z}$ of the promoter by a mechanism not involving cooperative binding (34). Therefore, the requirement for both proximal and distal $\mathrm{Z}$ binding sites in HeLa cells is unlikely to reflect cooperative binding but may be due to the ability of the transactivator domains of $\mathrm{Z}$ bound at proximal and distal sites to interact cooperatively. In Jurkat cells, the $Z$ 
A

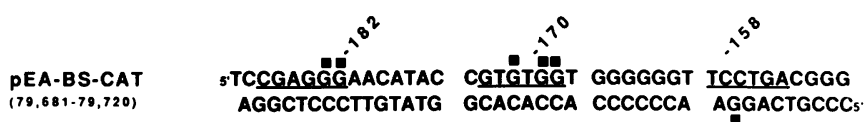

PEA- 182 -AGATCT-.

PEA- $\triangle 170$

--AGATCT--

PEA- $\triangle 158$

..TCTAGA.

B

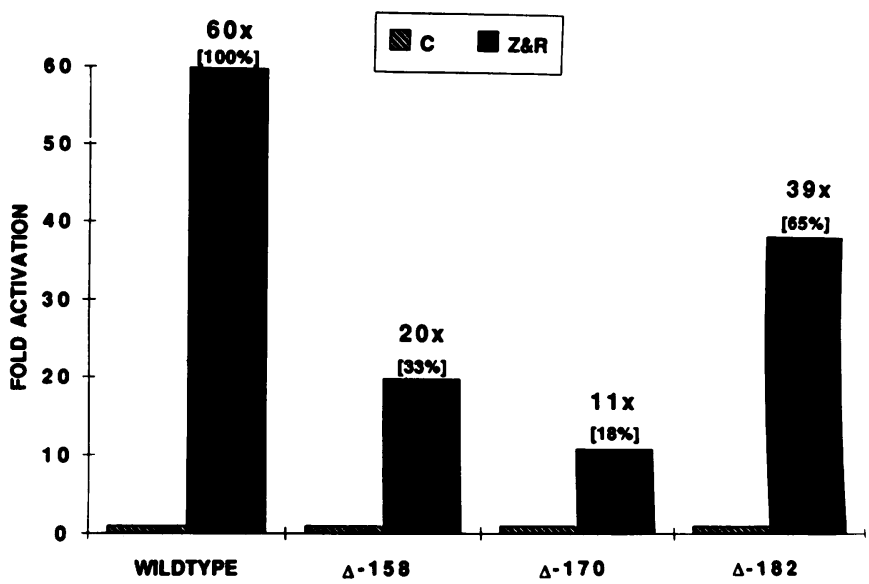

Figure 7. Construction of BMRF1 RRE site-directed mutants. $\square$ indicates a protected guanine residue, $\square$ indicates a weakly protected guanine residue. Regions of homology with other $\mathrm{R}$ binding sites are indicated by brackets on the parent construct, pEA-BS-CAT, which contains the EBV BamHI-RsaI fragment linked to CAT in a phagemid vector (25). Three regions (underlined) were abolished using the Kunkel method (31) of site-directed mutagenesis as shown. The new sequences of the mutant constructs are shown below the corresponding region of the parent construct.

transactivator alone cannot efficiently activate the BMRF1 promoter, but the combination of $\mathrm{Z}$ and $\mathrm{R}$ together produces a striking degree of activation (25). The presence of either ZRE alone was sufficient for cooperative transactivation by the Z-plus$\mathbf{R}$ combination in Jurkat cells. Thus, our findings suggest that the mechanism of Z-induced transactivation of early EBV promoters may vary from one cell type to another.

Two additional weak $Z$ binding sites were identified which did not contribute to $\mathrm{Z}$ responsiveness of the BMRF1 promoter in HeLa cells or Z-plus-R responsiveness in Jurkat cells. These sites may play a role in $\mathrm{Z}$-induced activation in another, as yet unidentified, context. Additionally, there may still be unidentified $\mathrm{Z}$ binding sites in the BMRF1 promoter with reduced binding affinity making identification of these sites very difficult.

The $R$ protein was only recently shown to be a sequencespecific DNA-binding protein. At present only three $R$ binding sites have been identified, two within the DR promoter and one within the early BMLF1 promoter (Fig. 10) $(21-24)$. The previously identified $\mathbf{R}$ binding sites have consisted of two distinct regions separated by 6-8 bases. Although all of the $R$ sites are GC-rich, each of the three sites have unique sequences. The

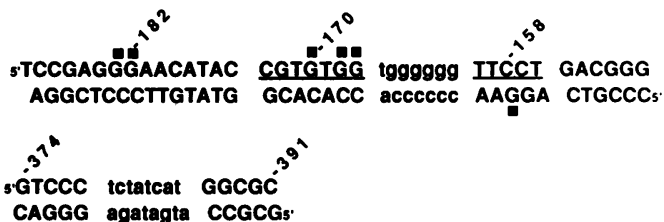

RRE-DR1

$(53,527.53,544)$

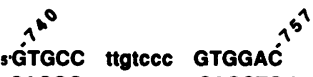

CACGG aacaggg CACCTGs'

RRE-DR2

$(53.549 .53,564)$

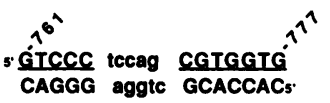

Figure 8. The BMRF1 sequence from -154 to -174 is required for $R$ binding A. A gel retardation assay with probes containing mutations in the $\mathbf{R}$ binding region of the BMRF1 promoter. Gel retardation assay was performed with the GR356 fusion protein and wildtype versus mutant BMRF1 probes spanning the -265 to -79 region. Mutant probes were isolated from the constructs shown in Fig. 7. Probes are indicated below each lane. The protein and the doublestranded oligomer used as competitor DNA at 1000 molar excess are indicated above each lane. (GR-: fusion protein constructed with $R$ in the antisense orientation; R: GR356 fusion protein; RRE-BMLF1: oligomer containing a BMLF1 promoter $\mathrm{R}$ binding site; AP-1: oligomer containing the AP-1 site in the BMRF1 promoter; RRE-BMRF1; oligomer containing the BMRF1 promoter region -153 to $-187 ; \mathrm{MYB}_{\mathrm{RE} 4}$ : oligomer containing four c-myb binding sites). B. Competition gel retardation assay with oligomers containing mutations in the $\mathrm{R}$ binding region of the BMRF1 promoter. A gel retardation assay was performed with GR356 fusion protein and a probe spanning the -265 to -79 region of the wildtype BMRF1 promoter. The protein and the double-stranded oligomer spanning the -153 to -187 region of the BMRF1 promoter used as competitor DNA at 1000 molar excess are indicated above each lane. (GR - : fusion protein constructed with $R$ in the antisense orientation; R: GR356 fusion protein; WT: wildtype RRE-BMRF1 oligomer; $\Delta-158$ : BMRF1 $\Delta-158$ oligomer; $\Delta-170$ : BMRF1 $\Delta-170$ oligomer; $\Delta-182$ : BMRF1 $\Delta-182$ oligomer.)

sequence in the region of the BMRF1 promoter bound by $R$ shows a site which is very similar to the DR-2 $R$ binding site located within the DR promoter. Given that $R$ was shown in this study to bind directly to the BMRF1 promoter, it is perhaps surprising that $R$ alone induces only a modest degree of activation of this promoter compared to the effect of $R$ upon the BMLF1 and DR promoters $(13,36)$. DR promoter sequences other than the actual $R$ binding sites have been shown to influence the ability of $R$ to activate the DR promoter (13). Therefore, it is possible that the location of the $\mathrm{R}$ binding site in the BMRF1 promoter is not favorable for $\mathrm{R}$ activation due to the proximity of a binding site for a cellular repressor of $\mathbf{R}$ activation or $\mathbf{R}$ binding.

Although we demonstrate that the -170 and -158 mutants can no longer bind $R$, these mutations retain a moderate level of R-induced activation (Fig. 9). We have observed previously that $\mathrm{R}$-induced transactivation may be mediated by more than one mechanism (37) and we have shown recently that the EBV BRLF1 promoter is moderately responsive to $R$ in transfection studies although $\mathrm{R}$ does not bind directly to this promoter (38). Therefore it is quite possible that the $\mathrm{R}$-induced activation of the BMRF1 promoter which persists after removal of the $R$ binding site results from a nonbinding mechanism of activation.

We have recently reported that the cellular transcription factor $\mathrm{c}-m y b$, like $\mathrm{R}$, induces cooperative transactivation of the BMRF1 promoter with $\mathrm{Z}$ in Jurkat cells but not HeLa cells. Unlike $\mathbf{R}$, c-myb does not bind directly to the BMRF1 promoter (26). The sequences surrounding the AP-1 site are sufficient to transfer Z- 


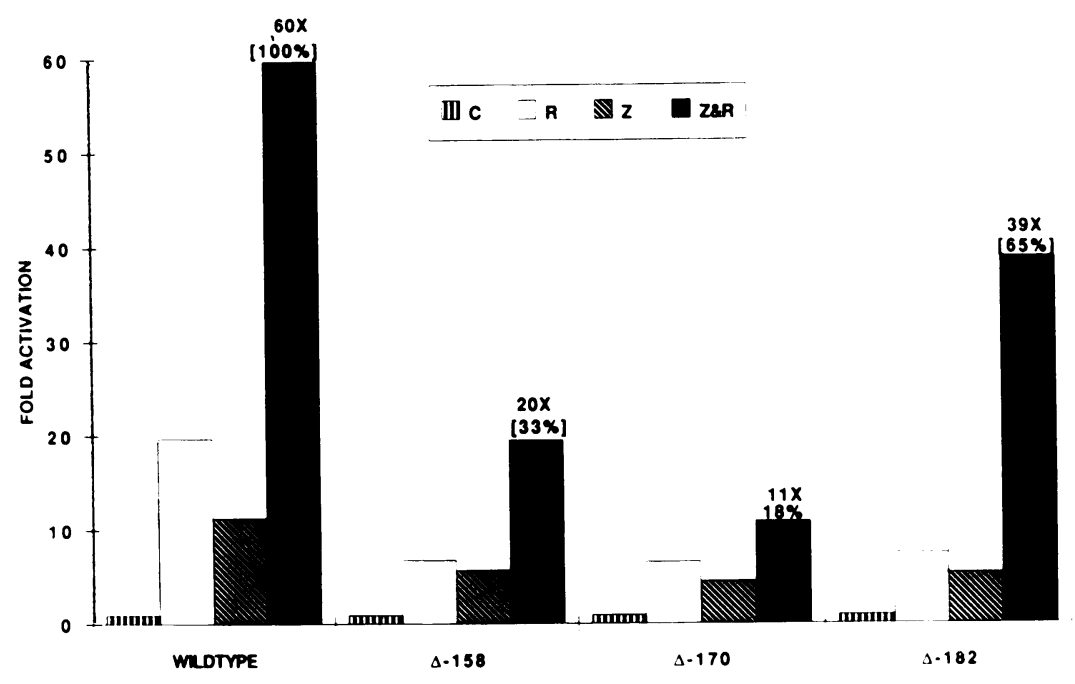

Figure 9. The $\mathrm{R}$ binding site is required for efficient Z-plus- $\mathrm{R}$ activation of the BMRF1 promoter in Jurkat cells. The CAT constructs pEA-BS-CAT (wildtype), pEA- $\Delta 158(\Delta-158)$, pEA- $\Delta 170(\Delta-170)$, or pEA- $\Delta 182(\Delta-182)$ were cotransfected with a control plasmid (四, pHD1013, C), pEBV-RIE ( $\square$, R), pEBV-ZIE ( Z), or both pEBV-ZIE and pEBV-RIE $(\mathbf{\square}, \mathrm{Z} \& \mathrm{R})$ into Jurkat cells. The results are presented as average fold activation of a minimum of seven experiments (indicated in the ordinate). The numbers above the bars indicate the average fold activation by the transactivator versus the control plasmid. The bracketed numbers above the Z\&R columns indicate the percent of the activity seen with the mutant promoter construct versus the intact (wildtype) promoter.

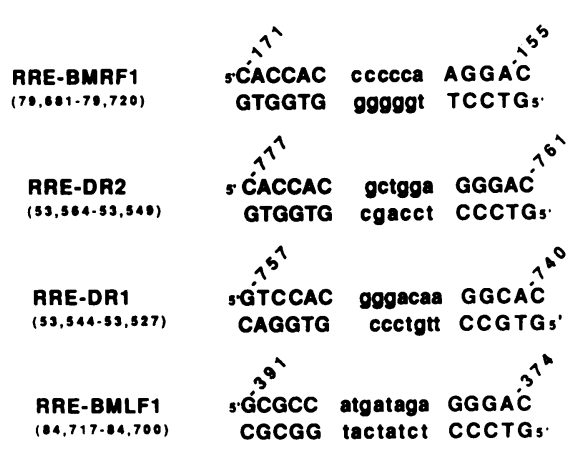

Figure 10. Comparison of $R$ binding sites. Numbers above the sequences indicate the region of the promoter in which the site is found. Numbers at the left indicate the region of the EBV genome in which these sites can be found.

plus-c-myb responsiveness to a heterologous promoter (26) but are not sufficient to transfer Z-plus- $R$ responsiveness (unpublished data). Therefore, it appears that Z-plus-R-induced activation of the BMRF1 promoter occurs through a different mechanism than the Z-plus-c-myb effect.

It is likely that cell-specific factors contribute to Z-plus-R cooperative activation of the BMRF1 promoter since this cooperation does not occur in epithelial cells. There are a number of ways that cellular factors may contribute to Z-plus-R cooperative activation. One possibility is that there is a BMRF1 promoter-specific inhibitor in lymphoid cells which prevents activation by one (or both) of these EBV transactivators. The binding of one of these EBV transactivator proteins to the BMRF1 promoter might disrupt binding of this hypothetical inhibitor and thereby increase the efficiency of the other EBV transactivator. Our finding that the AP-1 and -106 mutants have increased constitutive activity in Jurkat cells (more so than in HeLa cells, data not shown) suggests that lymphoid-specific inhibitors may bind near these regions. A second possibility is that there is a transactivator-specific inhibitor which inhibits either $\mathrm{Z}$ or $\mathrm{R}$ function in lymphoid cells and that this effect is abolished when both $\mathrm{R}$ and $\mathrm{Z}$ are bound to the BMRF1 promoter. In addition, a cellular factor present in HeLa cells but not in Jurkat cells may enhance cooperative interaction between the $\mathrm{Z}$ homodimers bound at the -106 and AP-1 sites.

At present complete understanding of the mechanism of transactivation by Z-plus-R is not possible. However, we have made a number of observations regarding Z-plus- $R$ cooperative activation. As described above, we found that both $Z$ and $R$ binding sites are required for cooperative activation by Z-plus$R$. To date a direct protein - protein interaction between $R$ and $\mathrm{Z}$ has not been demonstrated despite investigation by a number of laboratories (including our own). The lack of direct protein - protein interaction and the lack of Z-plus-R cooperative activation of the BMRF1 promoter in epithelial cells suggest that Z-plus- $R$ activation is dependent in some fashion on cell specific factors. These observations, along with observations made by other investigators (21), currently suggest that Z-plus-R cooperative activation is dependent upon 1) direct binding by $R$ and $\mathrm{Z}$ to responsive promoter elements and 2) interactions between $R, Z$ and cellular transcription factors.

\section{ACKNOWLEDGMENTS}

We are grateful to P.Farrell for the gift of the BZLF1 cDNA, Joseph S.Pagano for reviewing the manuscript and A.Baldwin for help with gel retardation assays. This work was supported by Public Health Service grants CA01229 and CA19014 from the National Institutes of Health, 500011-PG from the American Foundation of AIDS Research and MV-532 from the American Cancer Society. 


\section{REFERENCES}

1. Kieff, E. and Liebowitz, D. (1990) In Knipes, D.M. (eds.), Virology. Raven Press, Ltd, New York, 1889-1920.

2. Miller, G. (1990) In Knipes, D.M. (eds.), Virology. Raven Press, Ltd., New York, 1921-1958.

3. Chevallier-Greco, A., Manet, E., Chavrier, P., Mosnier, C., Daillie, J. and Sergeant, A. (1986) EMBO J., 5, 3243-3249.

4. Countryman, J. and Miller, G. (1985) Proc. Natl. Acad. Sci. USA, 82, $4085-4089$.

5. Gregory, C., Kirchgens, C., Edwards, C., Young, L., Rowe, M., Forster, A., Rabbits, T. and Rickenson, A. (1987) Eur. J. Immunol., 17, 1199-1207.

6. Grogan, E., Jenson, H., Countryman, J., Heston, L., Gradoville, L. and Miller, G. (1987) Proc. Natl. Acad. Sci. USA, 84, 1332-1336.

7. Miller, G., Rabson, M. and Heston, L. (1984) J. Virol., 50, 174-184.

8. Rooney, C., Taylor, N., Countryman, J., Jenson, H., Kolman, J. and Miller, G. (1988) Proc. Natl. Acad. Sci. USA, 85, 9801-9805.

9. Rooney, C.M., Rowe, D.T., Ragot, T. and Farrell, P.J. (1989) J. Virol., 63, 3109-3116.

10. Takada, K., Shimizu, N., Sakuma, S. and Ono, Y. (1986) J. Virol., 57, 1016-1022.

11. Farrell, P., Rowe, D., Rooney, C. and Kouzarides, J.T. (1989) EMBO J., 8, 127-132.

12. Flemington, E. and Speck, S. (1990) J. Virol., 64, 1227-1232.

13. Gruffat, H., Moreno, N. and Sergeant, A. (1990) J. Virol., 64, 2810-2818.

14. Kouzarides, T., Packham, G., Cook, A. and Farrell, P.J. (1991) Oncogene, 6, 195-204.

15. Lieberman, P.M., Hardwick, J.M., Sample, J., Hayward, G.S. and Hayward, S.D. (1990) J. Virol., 64, 1143-1155.

16. Sinclair, A.J., Brimmell, M., Shanahan, F. and Farrell, P.J. (1991) J. Virol., 65, 2237-2244.

17. Taylor, N., Flemington, E., Kolman, J.L., Bauman, R.P., Speck, S. and Miller, G. (1991) J. Virol., 65, 4033-4041.

18. Hardwick, J.M., Lieberman, P.M. and Hayward, S.D. (1988) J. Virol., 62, 2274-2284.

19. Manet, E., Gruffat, H., Trescol-Biemont, M., Moreno, N., Chambard, P., Giot, F. and Sergeant., A. (1989) EMBO J., 8, 1819-1826.

20. Cox, M.A., Leahy, J. and Hardwick, J.M. (1990) J. Virol., 64, 313-312.

21. Giot, J.F., Mikaelian, I., Buisson, M., Manet, E., Joab, I., Nicolas, J.C. and Sergeant, A. (1991) Nucleic Acids Res., 19, 1251-1258.

22. Gruffat, H., Duran, N., Buisson, M., Wild, F., Buckland, R. and Sergeant, A. (1992) J. Virol., 66, 46-52.

23. Gruffat, H., Manet, E., Rigolet, A. and Sergeant, A. (1990) Nucleic Acids Res., 18, 6835-6843.

24. Manet, E., Rigolet, A., Gruffat, H., Giot, J.F. and Sergeant, A. (1991) Nucleic Acids Res., 19, $2661-7$.

25. Holley-Guthrie, E.A., Quinlivan, E.B., Mar, E.-C. and Kenney, S. (1990) J. Virol., 64, 3753-3759.

26. Kenney, S.C., Holley-Guthrie, E.A., Quinlivan, E.B., Gutsch, D., Bender, T., Giot, J.-F. and Sergeant, A. (1992) Mol. Cell. Biol., 12, 136-146.

27. Lin, Y.-S., Carey, M.F., Ptashe, M. and Green, M. (1990) Nature, 345, $359-361$.

28. Toneguzzo, F., Hayday, A. and Keating, A. (1986) Mol. Cell. Biol., 6, $703-706$.

29. Davis, M.G., Kenney, S., Kamine, J., Pagano, J.S. and Haung., E.S. (1987) Proc. Natl. Acad. Sci. USA, 84, 8642-8646.

30. Gorman, C.M., Moffat, L.F. and Howard., B.H. (1982) Mol. Cell. Biol., 2, 1044-1051.

31. Kunkel, T.A. (1985) Proc. Natl. Acad. Sci. USA, 82, 488-492.

32. Bradford, M.M. (1976) Anal. Biochem., 72, 248-254.

33. Chodosh, L.A., Carthew, R.W. and Sharp, P. (1986) Mol. Cell. Biol., 6, 4723-4733.

34. Carey, M., Kolman, J., Katz, D.A., Gradoville, L., Barberis, L. and Miller, G. (1992) J. Virol., 66, 4803-4813.

35. Packham, G., Economou, A., Rooney, C.M., Rowe, D.T. and Farrell, P.J. (1990) J. Virol., 64, 2110-6.

36. Kenney, S., Holley-Guthrie, E., Mar, E.C. and Smith, M. (1989) J. Virol., 63, 3878-3883.

37. Quinlivan, E.B., Holley-Guthrie, E., Mar, E.-C., Smith, M.S. and Kenney, S. (1990) J. Virol., 64, 1817-1820.

38. Zalani, S., Holley-Guthrie, E.A., Gutsch, D. and Kenney, S.C. (1992) J. Virol., 66, 7282-7292. 